\title{
Femoral Hernioscopy: A Minimally Invasive Procedure to Evaluate Bowel Viability
}

\author{
Shafquat Zaman ${ }^{1}$, Mona Kamal ${ }^{2}$, Peter Waterland ${ }^{3}$
}

\begin{abstract}
We highlight an important surgical technique that can be utilized to prevent unnecessary laparotomies in patients undergoing emergency femoral hernia repair. A 79-year-old female presented to our unit with an increasingly painful left-sided groin swelling. She was subsequently taken to the operation theater for an emergency operation and found to have a spontaneously reduced femoral hernia after induction of anesthesia. Femoral hernioscopy was performed successfully with the patient positioned in a steep Trendelenburg position. This allowed us to perform a diagnostic laparoscopy and assess bowel viability. Consequently, further unnecessary procedures were avoided together with their associated short- and long-term morbidity. Hernioscopy is a safe, feasible, and valuable technique that is potentially underutilized. We feel that this is a cornerstone in the application of minimal access surgery for a common general surgical emergency, and hence further research with application of this technique is required in this field.

Keywords: Femoral hernia, Hernioscopy, Laparoscopy, Laparotomy.

World Journal of Laparoscopic Surgery (2019): 10.5005/jp-journals-10033-1378
\end{abstract}

\section{Case Description}

A 79-year-old lady was referred from primary care with an increasingly painful left groin swelling over several months. Her past surgical history included a left-sided total hip replacement. Clinical examination revealed a $3 \times 3 \mathrm{~cm}$ hard, fixed swelling below and lateral to the pubic tubercle. This was extremely tender on palpation.

An ultrasound of the left groin showed a $2.6 \mathrm{~cm}$ thick-walled cystic structure with no changes noted on the Valsalva maneuver. There was no free fluid or discrete collections seen on imaging. The patient was subsequently taken to the theatre for groin exploration.

\section{Surgical Technique}

A left-sided groin crease incision was made, and a femoral hernia identified. The hernia sac contained hemoserous fluid only. In order to further inspect the retracted bowel, a blunt $12 \mathrm{~mm}$ balloon port was introduced via the hernial sac into the abdominal cavity (Fig. 1). Standard insufflation with carbon dioxide was performed, maintaining an intra-abdominal pressure of $14 \mathrm{~mm} \mathrm{Hg}$. The patient was repositioned in a steep Trendelenburg position, and pneumoperitoneum was established.

Laparoscopy showed a mildly congested but viable small bowel loop. Constriction markings were seen on this segment of the bowel and were consistent with entrapment within the hernia sac (Fig. 2). As the loop of bowel was completely viable, no resection was required. The laparoscope was withdrawn, the port was removed, and a standard mesh repair of femoral hernia was performed. Postoperatively the patient made an excellent recovery and was discharged home the following day.

\section{Discussion}

Femoral hernia is the protrusion of a peritoneal sac through the femoral ring into the femoral canal. Less common than inguinal hernias, it is seen most frequently in older females. It is associated with higher rates of acute complications such as incarceration and strangulation.

\footnotetext{
${ }^{1-3}$ Department of General Surgery, Russells Hall Hospital, Dudley, West England, UK
}

Corresponding Author: Shafquat Zaman, Department of General Surgery, Russells Hall Hospital, Dudley, England, UK, Phone: +91 7944657005, e-mail: shafquatzaman@nhs.net

How to cite this article: Zaman S, Kamal M, Waterland P. Femoral Hernioscopy: A Minimally Invasive Procedure to Evaluate Bowel Viability. World J Lap Surg 2019;12(3):133-134.

Source of support: Nil

Conflict of interest: None

Femoral hernioscopy is a particularly useful technique in the emergency setting as it can potentially avoid a further explorative laparotomy. It allows the contents of a spontaneously reduced hernia to be fully inspected.

It is relatively quick to perform (approximately 10 minutes), and the technique can be adapted depending on the clinical context such as inguinal sac hernioscopy. In our case, a single port, in combination with the Trendelenburg position, provided excellent views of the reduced hernial sac contents. A single incision laparoscopic port (SILS) can also be used to allow the introduction of further instruments to obtain better views.

There are limited reports in the literature especially, on femoral hernioscopy as a technique for evaluating incarcerated hernias that retract under anesthesia. One study involving five patients with inguinal and femoral hernias showed that the technique was simple and may prevent unnecessary laparotomies. ${ }^{1}$ Kneessy and Weinbaum ${ }^{2}$ described a similar approach undertaken to examine a loop of incarcerated bowel, which had spontaneously retracted back into the abdomen. It appears that their patient positioning was not modified, and so an additional $5 \mathrm{~mm}$ port was required to manipulate and handle bowel.

Valderrama et al. ${ }^{3}$ used a smaller single $5 \mathrm{~mm}$ port for the insertion of a $0^{\circ}$ laparoscope in the management of an incarcerated right femoral hernia causing small bowel obstruction.

(-) The Author(s). 2019 Open Access This article is distributed under the terms of the Creative Commons Attribution 4.0International License (https://creativecommons. org/licenses/by-nc/4.0/), which permits unrestricted use, distribution, and non-commercial reproduction in any medium, provided you give appropriate credit to the original author(s) and the source, provide a link to the Creative Commons license, and indicate if changes were made. The Creative Commons Public Domain Dedication waiver (http://creativecommons.org/publicdomain/zero/1.0/) applies to the data made available in this article, unless otherwise stated. 


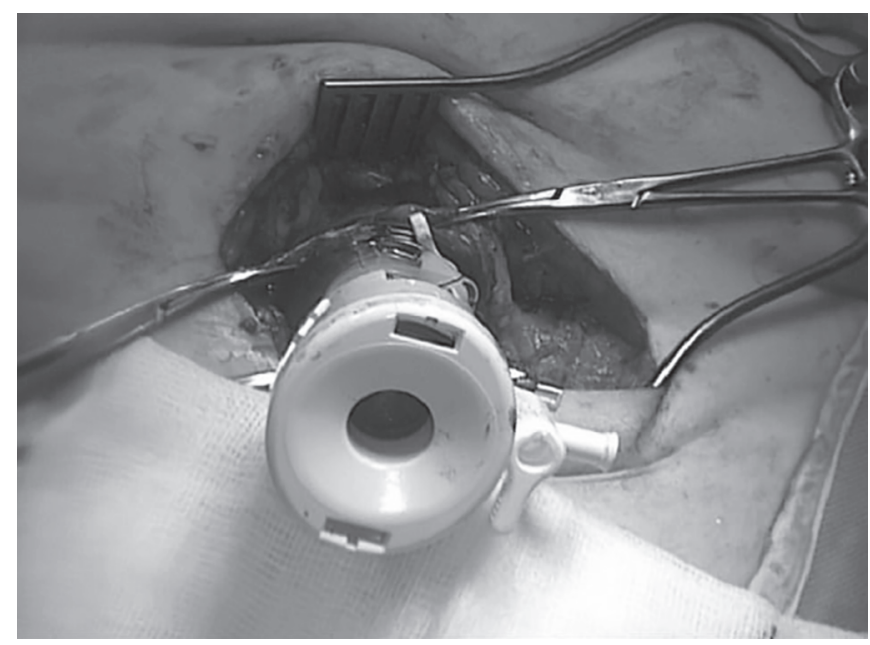

Fig. 1: $12 \mathrm{~mm}$ laparoscopic port inserted into the peritoneal cavity via the femoral canal

They demonstrated that this was a safe and useful technique in the treatment of such cases.

Romain et al. ${ }^{4}$ assessed the prognostic factors associated with postoperative morbidity and mortality following strangulated groin hernia repairs. They compared explorative laparotomy, laparoscopy, and hernioscopy and noted that explorative laparotomy was a major cause of postoperative complications.

Potential drawbacks of this technique include inadequate mobilization of bowel necessitating the introduction of additional ports. However, this problem can be addressed by the use of a SILS port. In addition, in some cases, views may be obscured in the presence of dense intra-abdominal adhesions or gross ascites.

Nevertheless, hernioscopy without additional trocar placement is a valuable technique that can be utilized by skilled and experienced laparoscopic surgeons to prevent unnecessary laparotomies. ${ }^{4,5}$ This will help to reduce the many complications associated with major surgery. Amongst the benefits include reduced risk of infection, postoperative pain, length of stay, wound dehiscence, incisional hernias, earlier mobilization, and reduced thromboembolic events.

In addition, inspection via the laparoscope may provide additional useful information such as the presence of ascitic fluid or tumors.

\section{Conclusion}

In situations where the contents of a hernial sac have reduced spontaneously under anesthesia prior to inspection, we advocate the use of hernioscopy. In skilled hands, the technique is safe

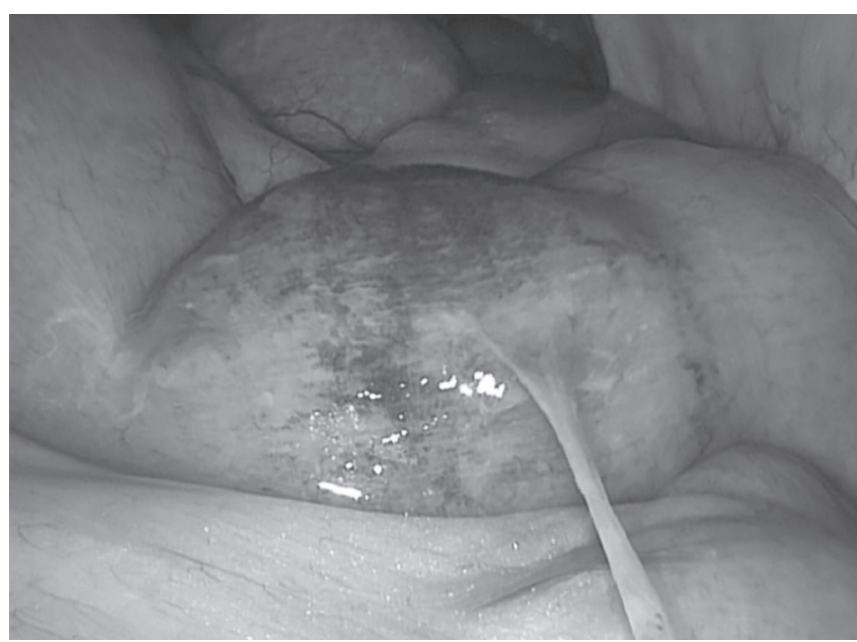

Fig. 2: Laparoscopy showing a segment of entrapped small bowel with visible constriction markings

and simple and can prevent unnecessary laparotomies and their associated complications.

\section{Clinical Significance}

There have not been sufficed case reports on this technique for a formal case series to be undertaken to look into the precise complications of femoral hernioscopy postoperative and long-term follow-up. We feel this is a cornerstone in application of minimal access surgery for a common general surgical emergency, and hence further research with the application of this technique is required in this field.

\section{References}

1. Morris-Stiff G, Hassn A. Hernioscopy: a useful technique for the evaluation of incarcerated hernias that retract under anesthesia. Hernia 2008;12(2):133-135. DOI: 10.1007/s10029-007-0296-2.

2. Kneesy K, Weinbaum F. Hernioscopic retrieval of bowel for evaluation of viability during repair of a Richter's-type incarcerated femoral hernia. Surg Laparosc Endosc 1997;7(2):171-172. DOI: 10.1097/00019509-199704000-00023.

3. Valderrama AR, Ruiz D, Malik M, et al. Femoral hernia sac laparoscopy: a case report. Minim Invasive Ther Allied Technol 2014;23(1):55-57. DOI: 10.3109/13645706.2013.831108.

4. Romain B, Chemaly R, Meyer N, et al. Prognostic factors of postoperative morbidity and mortality in strangulated groin hernia. Hernia 2012;16(4):405-410. DOI: 10.1007/s10029-012-0937-y.

5. Lin E, Wear K, Tiszenkel HI. Planned reduction of incarcerated groin hernias with hernia sac laparoscopy. Surg Endosc 2002;16(6):936-938. DOI: $10.1007 / \mathrm{s} 00464-001-8242-3$. 